\title{
Winners of the 2019 JA Ōmura Awards for excellence
}

\author{
Richard E. Lee ${ }^{1} \cdot$ Minoru Yoshida ${ }^{2}$
}

Received: 31 August 2020 / Accepted: 31 August 2020 / Published online: 27 October 2020

(c) The Author(s), under exclusive licence to the Japan Antibiotics Research Association 2020

The 2019 winner of the Ōmura Award for original articles goes to the authors of two consecutive outstanding papers on discovery of a novel antifungal antibiotic [1, 2], entitled "Discovery of a new antifungal agent ASP2397 using a silkworm model of Aspergillus fumigatus infection" by Ikuko Nakamura, Ryuichi Kanasaki, Koji Yoshikawa, Shigetada Furukawa, Akihiko Fujie, Hiroshi Hamamoto and Kazuhisa Sekimizu and "ASP2397: a novel antifungal agent produced by Acremonium persicinum MF-347833” by Ikuko Nakamura, Seiji Yoshimura, Teruhisa Masaki, Shigehiro Takase, Keisuke Ohsumi, Michizane Hashimoto, Shigetada Furukawa and Akihiko Fujie.

Invasive pulmonary aspergillosis is a life-threatening disease caused by infection with the fungus A. fumigatus. The incidence of invasive pulmonary aspergillosis is increasing among transplant patients or patients suffering from neutropenia and is a major cause of mortality among the immunocompromised patients. Unfortunately, the clinical efficacy of existing antifungal drugs remains quite poor. In order to find structurally unique drug candidates effective in vivo, the research group led by Nakamura utilized a silkworm larvae model of infection to evaluate the antifungal activity of a library of natural products derived from numerous fungal species isolated from geographically diverse tropical regions. As a result, the authors discovered ASP2397 and its derivatives from the culture broth of Acremonium persicinum MF-347833, which protected not only silkworm larvae but also mice from lethal infection with A. fumigatus [1]. Surprisingly, the chemical structure of ASP2397 determined in the subsequent paper [2] showed close similarity to that of ferrichrome, a fungal siderophore

Richard E. Lee

Richard.Lee@STJUDE.ORG

Minoru Yoshida

yoshida@riken.jp

1 St. Jude Children's Research Hospital, Memphis, TN, USA

2 RIKEN Center for Sustainable Resource Science, Wako, Saitama, Japan with high specificity for iron. ASP2397 is a cyclic hexapeptide chelating $\mathrm{Al}$ instead of Fe. The metal free form of ASP2397 (AS2488059) can chelate not only Al but also Fe and $\mathrm{Ga}$, although the Fe-chelating form (AS2488053) was less active than ASP2397. Recently, sideromycins, a group of antibiotics linked to siderophores by covalent bonding, have attracted much attention in developing antimicrobial agents, because siderophores are actively transported into cells for uptake of iron, which enables the antibiotic to reach its intracellular target. ASP2397 is a novel type of sideromycin, which constitutes a structurally mimic of the siderophore itself and is transported into Aspergillus cells via siderophore iron transporter (Sit1) [3].

ASP2397 is a clinically promising antifungal antibiotic as it showed strong cytotoxicity not only to A. fumigatus but also azole- and echinocandin-resistant Candida glabrata. Currently, ASP2397 (renamed VL-2397) is under development as a clinical antifungal drug by Vical Pharmaceuticals. Phase I clinical trials demonstrated that it was well tolerated in healthy volunteers and showed no serious adverse events even at high doses. The FDA granted fast track designation to this exciting antifungal drug candidate for the treatment of invasive aspergillosis.

The 2019 winner of the Ōmura Award for a review article is "Bacterial proteases, untapped antimicrobial drug targets" by Elizabeth Culp and Gerard D. Wright [4]. Bacterial proteases represent a large, under-exploited class of novel therapeutic targets for antibiotic development. In contrast to other areas of human medicine, where protease inhibitors have great utility as antiviral agents and for the treatment of hypertension, there has been a noticeable lack of development against these targets in the antibacterial space. This review provides a mechanistic overview of protease action, focusing on intracellular proteolytic complexes (Lon, HslUV, ClpXP and $\mathrm{FtsH}$ ) and type I/II signal peptidases as potential targets.

Known inhibitors of these enzymes are carefully reviewed, most of natural product origin, indicating the ligand-ability of these targets. Chemical modulation of the Clp system, the most extensively studied intracellular proteolytic complex targeted to date, is examined in detail. The 
utility of inhibitors and activators of ClpP, and uncouplers of the associated AAA + ATPases ClpC and ClpX are highlighted. The challenges associated with the development of antibacterial protease therapeutics are also noted, which include: low selectivity due to close homology to their human protease counterparts, particularly those found in the mitochondria; a propensity for spontaneous resistance development, as many of these targets are not essential; and obtaining entry of activators and inhibitors into Gramnegative bacteria and Mycobacterium tuberculosis.

With a focus on AAA + family proteolytic complexes and signal peptidases, this outstanding review provides an informative introduction to bacterial proteases and provides a full picture of the therapeutic potential of these targets The subject matter is timely, as there is a clear need for new antibacterial agents that act against alternative molecular targets to overcome resistance to current classes of antibacterial drugs.

\section{References}

1. Nakamura I, Kanasaki R, Yoshikawa K, Furukawa S, Fujie K, Hamamoto H, et al. Discovery of a new antifungal agent ASP2397 using a silkworm model of Aspergillus fumigatus infection. J Antibiot. 2017;70:41-4.

2. Nakamura I, Yoshimura S, Masaki T, Takase S, Ohsumi K, Hashimoto M, et al. ASP2397: a novel antifungal agent produced by Acremonium persicinum MF-347833. J Antibiot. 2017;70: $45-51$.

3. Nakamura I, Ohsumi K, Takeda S, Katsumata K, Matsumoto S, Akamatsu S, et al. ASP2397 is a novel natural compound that exhibits rapid and potent fungicidal activity against Aspergillus species through a specific transporter. Antimicrob Agents Chemother. 2019;63:e02689-18.

4. Culp E, Wright GD. Bacterial proteases, untapped antimicrobial drug targets. J Antibiot. 2017;70:366-77.

\section{ABOUT THE WINNERS}

The medal will be awarded to the first author of original and review articles, and all of the authors will receive the certificates. Each first author's short biography is as follows.

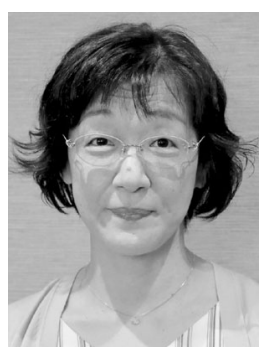

Original article division; awardee Ikuko Nakamura Ikuko Nakamura is a pharmacologist specializing in the field of fermentation drug development utilizing secondary metabolites. She received her B.S. (1991) in Pharmaceutical Sciences at Tohoku University and was engaged in drug discovery from natural products after entering Fujisawa Pharmaceutical Co., Ltd. (currently known as Astellas Pharma Inc.). She has been mainly working on creating new antifungal candidate compounds. She established silkworm A. fumigatus infection model (2004) under the advice of Prof. Kazuhisa Sekimizu in Pharmaceutical Sciences at the University of Tokyo and discovered novel antifungal agent (ASP2397). After the development research of ASP2397, she worked on the development of antiallergic agent as a senior researcher at Astellas until 2019. She received her Ph.D. (2020) from Tohoku University under the supervision of Prof. Yoshiharu Iwabuchi. She is currently raising her child.
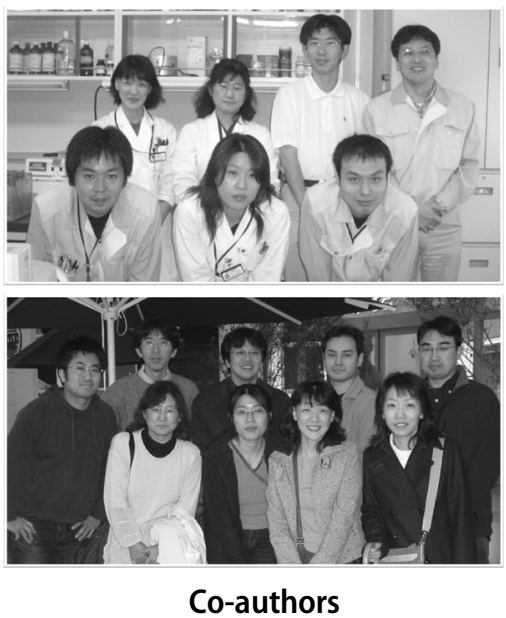

Review division; awardee Elizabeth Culp Elizabeth Culp received her BSc from McMaster University in 2015, majoring in biology. Under the supervision of Dr. Bhagwati Gupta, her undergraduate thesis involved applying CRISPR/Cas9 technology to nematodes. Continuing her doctoral studies at McMaster University in biochemistry and biomedical science, she is currently working toward her Ph.D. under the supervision of Dr. Gerry Wright. Her research interests revolve around using biosynthetic gene clusters to guide the rational discovery of antibiotics from Actinomycetes. Approaches falling under this common theme included inactivation of nuisance biosynthetic gene clusters, evolution-guided gene cluster prioritization, and bacterial protease targeting natural products, the focus of this review article. 